\title{
SUPERCONDUCTING CRITICAL TEMPERATURE OF LAYERED MERCUROCUPRATE FAMILY
}

\author{
A.L. KuzemskY ${ }^{a}$, I.G. Kuzemskaya ${ }^{b}$ AND A.A. Cheglokov ${ }^{a}$ \\ ${ }^{a}$ Bogolyubov Laboratory of Theoretical Physics, Joint Institute for Nuclear Research \\ 141980 Dubna, Moscow Region, Russia \\ ${ }^{b}$ High Pressure Physics Institute, Russian Academy of Sciences \\ 142092 Troitsk, Moscow Region, Russia
}

\begin{abstract}
We study the physics of the mercurocuprate family from the structural point of view. We present a phenomenological approach determining the critical temperatures for homologous series $\mathrm{HgBa}_{2} \mathrm{Ca}_{n-1} \mathrm{Cu}_{n} \mathrm{O}_{2 n+2+\delta}$ depending on the number of layers $n$. The model is based on the Lawrence-Doniach theory of layered superconductors modified for the case of inequivalent layers. The redistribution of charge was taken into account. This leads to observable nonmonotonic "bell"-shaped dependence of $T_{\mathrm{c}}(n)$ and provides a quantitative explanation of the experiments.
\end{abstract}

PACS numbers: 74.25.Nf, 74.72.Bk, 74.72.Gr

There is considerable current interest in the properties of superconducting copper oxides. Particular importance is the question of interrelation of crystal structure, superstructure, superconductivity, and the role of anisotropy in layered superconducting cuprates. Mercurocuprates have a pronounced layered structure and have raised once again the problem of how a structure of these materials affects their properties. The homologous series $\mathrm{HgBa}_{2} \mathrm{Ca}_{n-1} \mathrm{Cu}_{n} \mathrm{O}_{2 n+2+\delta}$ is of special interest because it culminates the fascinating features of high $-T_{c}$ superconductors (HTSC) and are still most high- $T_{\mathrm{c}}$ representatives of cuprates [1].

One of the most important issue for mercurocuprate family is to understand the exact role of layered structure and main parameters that govern the highest value of transition temperature of these materials [2].

Recently, there has been great progress in synthesis of high quality samples and detailed structural studies. The structure of the family of mercurocuprates can be viewed as consistent of $\mathrm{Ca}_{n-1} \mathrm{Cu}_{n} \mathrm{O}_{2 n}$ block and the $\mathrm{Hg}-\mathrm{O}_{\delta}$ block which play a role of reservoir of charge. The fabricating of homologous series $\mathrm{HgBa}_{2} \mathrm{Ca}_{n-1} \mathrm{Cu}_{n} \mathrm{O}_{2 n+2+\delta}$ has been performed using the high-pressure high-temperature synthesis which seems to be the efficient and workable method [3] to produce high quality $\mathrm{Hg}$-superconducting samples. The synthesis of $n=1,2, \ldots 7$ of the $\mathrm{Hg}$-based homologous series was performed by this and other techniques with $T_{\mathrm{c}}$ listed in Table. 
TABLE

Literature data.

\begin{tabular}{c|c|c|r}
\hline \hline$n$ & $T_{\mathrm{c}}$ & $\delta$ & $a$ \\
\hline 1 & $97-98$ & 0.08 & 3.880 \\
2 & 127 & $0.22(4)$ & 3.8580 \\
3 & 135 & $0.28-0.35$ & 3.852 \\
4 & $125-126$ & $0.4(1)-0.46$ & 3.847 \\
5 & $110-114$ & $0.32(3)$ & 3.8523 \\
6 & $96-100$ & - & 3.8533 \\
7 & 88 & - & -
\end{tabular}

The concept of a homologous series [1], which in the case of mercurocuprates plays an especially important role, raise the natural question about dependence of a superconducting critical temperature of this family of layered copper oxides from the number of pairs $(n-1)$ of $\mathrm{CuO}_{2}$ and bare cation planes in the infinite layer block. In other words, because one member of one homologous series has the same charge-reservoir block [1] but the number of $\mathrm{CuO}_{2}$ planes $(n)$ is different, the main interest is dependence $T_{c}(n)$ [4]. There seems to be a close relationship between the average copper valence and the phase produced in the high-pressure synthesis of mercurocuprates. The formal copper valence for different members of mercurocuprate family is equal to $v_{\mathrm{Cu}}=2(n+\delta) / n$. The values of $\delta$ obtained from neutron scattering experiments lead to conclusion that the extra oxygen content, and consequently the copper valence and lattice parameter $a$ depend both on the number of $\mathrm{CuO}_{2}$-layers and heat treatment of the samples. There are certain evidences that as regards to the inequivalent $\mathrm{CuO}_{2}$ layers in multilayer structure the inner and outer $\mathrm{CuO}_{2}$ layers can have different charge carrier density. Thus the correlation between the copper valence, lattice parameters, and extra oxygen contents becomes then very important.

The workable phenomenological approach for calculation of transition temperature of layered cuprates has been proposed in Ref. [5]. The physical idea was that there are distinct "active" elements in the multilayer structure. To each active element denoted $l$ in the structure was associated a Ginsburg-Landau (GL) order-parameter field $\psi_{l}(r)$. Each of these $\psi_{l}(r)$ has some distinct "bare" transition temperature $T_{l}^{0}$ in that structure. The full GL theory is a generalization to coupling order parameters of the work of Lawrence and Doniach.

In this report we apply this line of reasoning for the mercurocuprate family. The free energy density was taken in Ref. [5] as a bilinear form in the spacially independent order parameters

$$
f=\sum_{i} a_{i}(T)\left|\psi_{i}\right|^{2}+\sum_{i j} v_{i j}\left|\psi_{i}-\psi_{j}\right|^{2}
$$

here $v_{i j}$ is the coupling coefficient. This leads to relevant secular equation of the form

$$
\left\|\left(a_{i}(T) n v_{i j}\right) \delta_{i j}-v_{i j}\right\|=0 .
$$


According to Ref. [5], the transition temperature for an $n$-layer sample is determined as the maximum eigenvalue of the $n$-dimensional tridiagonal matrix. It is possible to consider a system composed of $n$ identical layers, where all parameters are identical $\left(T_{l}^{0}=T^{0}\right.$ and $\left.v_{i j}=v\right)$. For this case the explicit expression [5] for $T_{\mathrm{c}}$, provided that the largest solution is $T=T^{0}, T_{\mathrm{c}}(n)$ is an increasing function of $n$ with upper limit $T_{\mathrm{c}}(n=\infty)=141 \mathrm{~K}$. In this report we use the structural model of a periodic layered system, whose unit cell contains two kinds ( $a$ and $b$ ) of layers ("active" elements) $(1 \leq a \leq n$ and $1 \leq b \leq m)$. Equation (3) is identical to the following expression:

$$
\operatorname{det}(T I-F)=0, \quad F_{i j}=\left(T_{i}^{0}-\frac{v_{i-1, i}}{a_{i}^{0}}-\frac{v_{i, i+1}}{a_{i}^{0}}\right) \delta_{i j}+\frac{v_{i j}}{a_{i}^{0}} \delta_{i, j \pm 1} .
$$

Equations (3) and (4) properly determine the critical temperature as a maximum solution $T_{i}^{\max }$ of this periodic $n-m$ layered system. The numerical solution of these equations with reasonable parameters show that $T_{\mathrm{c}}$ of the whole system is raised with the number $n$ and reduced with the number $m$. This reflects the fact that the amplitudes of the order parameters for $a$-layers are larger than those for $b$-layers. This interplay of the two order parameters of the two kinds of active elements is one of the most important properties of the present phenomenological considerations. Nevertheless, the model properties related to these effects do not give the observable "bell"-shaped form of $T_{\mathrm{c}}(n)$. It is therefore necessary to take into account the interlayer effects of charge redistribution to fit the experimental data. It was supposed that in HTSC a charge redistribution may occur between the reservoir block and active block under various conditions like applied high pressure or with changing of composition, such as oxygen stoichiometry. The inequivalent layer models reveal essential feature arising from the sensitivity to the transition of one of the subsystems to the superconducting state so that $n_{h}^{l}$ should rearrange when the system becomes superconducting. We adopt the following workable ansatz for the critical temperature $T_{a}$ of an $a$-layer dependence on the number density of carriers of the form:

$$
T_{a}\left(x_{c}\right)=T_{a}^{0}\left[1-\left(\frac{x_{c}-h_{2}}{h_{1}}\right)^{2}\right]^{1 / 2},
$$

where the carrier-number density $x_{c}$ in a single layer is given by $x_{c}$ $=x_{\text {out }}^{b}[m c+2(1-c)] /(n+m)$ and $x_{\text {out }}^{b}$ is the fraction of carriers which is provided by the outer $b$-layer adjacent to an $a$-layer per area while $x_{\text {in }}^{b}=c x_{\text {out }}^{b}(c \leq 1)$ is the corresponding quantity provided by an inner $b$-layer. Here $h_{1}$ and $h_{2}$ are fitting parameters. To proceed further we use the following model estimations:

$$
\begin{aligned}
& T_{b}= \begin{cases}T_{b}^{0}\left(x_{c} / x_{0}\right), & 0 \leq x_{c} \leq x_{0}, \\
T_{b}^{0}, & x_{c} \geq x_{0},\end{cases} \\
& a_{b}^{0}\left(x_{c}\right)= \begin{cases}a_{b} 0 T_{b}^{0}\left(x_{c} / x_{0}\right)^{1 / 2}, & 0 \leq x_{c} \leq x_{0}, \\
2 a_{b} 0 T_{b}^{0} \frac{x_{0}}{x_{c}+x_{0}}, & x_{c} \geq x_{0} .\end{cases}
\end{aligned}
$$

Here $x_{0}=m^{*} t_{\perp} / \pi \hbar^{2}$ and $v=v_{i j}$ have no $x_{c}$ dependence. The quantities $m^{*}$ and $t_{\perp}$ were taken from band structure calculations. With these simplifications it 
is possible now to calculate the critical temperature. There are the following free model parameters:

(i) $n$ and $m$ which determine the structure,

(ii) $x_{\text {out }}^{b}$ and $c$ which determine $x_{c}$,

(iii) $x_{0}$ and $T_{b}^{0}$ which determine $T_{b}$,

(iv) $a_{a}^{0}, a_{b}^{0}$, and $v$.

We have calculated $T_{c}(n)$ with $m$ fixed for $m=1,2,3,4$. The results of numerical calculations for $T_{\mathrm{c}}(n)$ for $m=3$ (Fig. 1) are shown as a broken solid line. Thus, incorporating the charge interlayer redistribution, it is possible to restore the observable "bell"-shaped dependence even in the simplest version of the model and fit the experimental results well.

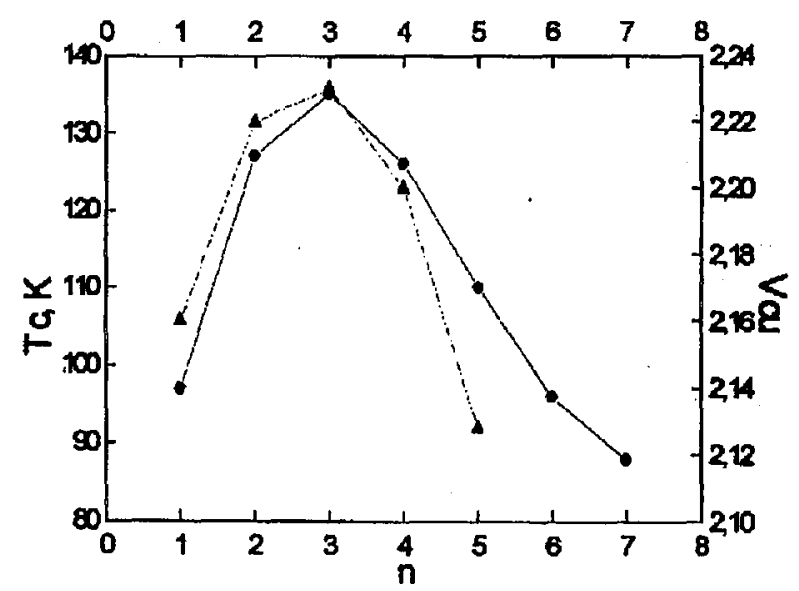

Fig. 1. Dependence of the superconducting critical temperature $T_{c}$ and copper valence $v_{\mathrm{Cu}}$ on the number of layers $n$. Full line is the critical temperature and dashed line is valence. Dependence of $T_{c}(n)$ was calculated with $m=3$ fixed. The points are the experimental data. Parameters: $T_{a}^{0}=189.1, T_{b}^{0}=88.1, h_{1}=0.313, h_{2}=0.504$.

In summary, in this paper we have presented a simple phenomenological model which rationalizes reasonably the currently available experimental data for the mercurocuprate family.

\section{References}

[1] H. Yamauchi, M. Karppinen, S. Tanaka, Physica C 263, 146 (1996).

[2] M. Karppinen, H. Yamauchi, J. Supercond. 11, 39 (1998).

[3] K.A. Lokshin, I.G. Kuzemskaya, L.F. Kulikova, E.V. Antipov, E.S. Itskevich, Physica C 279, 11 (1997).

[4] J. Karpinski, H. Schwer, E. Kopnin, R. Molinski, K. Konder, J. Hofer, D. Zech, J. Supercond. 11, 119 (1998).

[5] J. Birman, J.P. Lu, Phys. Rev. B 39, 2238 (1989). 